\title{
Effect of diameter on force generation and responsiveness of bronchial segments and rings
}

\author{
P.R. Gray, H.W. Mitchell
}

Effect of diameter on force generation and responsiveness of bronchial segments and rings. P.R. Gray, H.W. Mitchell. (CERS Journals Ltd 1996.

ABSTRACT: In this study, isovolumic bronchial segments and bronchial rings were used to investigate the influence of airway diameter on smooth muscle force generation and acetylcholine responsiveness.

Segments with internal diameters ranging from 1.0-6.0 mm were obtained from the mainstem bronchus of eight pigs. Responses to increasing acetylcholine concentrations were quantified in segments by intralumenal pressure $\left(\mathrm{cmH}_{2} \mathrm{O}\right)$, and in rings by tension $\left(\mathrm{g}^{\mathrm{cm}}-\mathbf{1}\right)$. The negative $\log$ of the concentration producing half the maximal effect (EC50) (i.e. $\mathrm{pD}_{2}$ ) to acetylcholine was calculated for each segment and ring. Ring tension was used to calculate a theoretical lumen pressure for each ring, and this, along with the $\mathrm{pD}_{2}$, was compared with values obtained from segments of the same diameter.

Intermediate-sized segments produced significantly greater intralumenal pressures than did large or small segments. Small segments were 160 times more sensitive to acetylcholine than large segments. In contrast to the segments, bronchial rings showed no effect of size on acetylcholine sensitivity. Theoretical ring lumen pressures matched those measured for large and intermediate segments, but not for small segments.

The different behaviour of bronchial segments and rings obtained from the same sized airway suggests that the three-dimensional architecture of the airway is an important factor in determining behaviour, particularly in small airways. Eur Respir J., 1996, 9, 500-505.
Dept of Physiology, The University of Western Australia, Nedlands, WA, Australia.

Correspondence: P.R. Gray

Physiology Dept

The University of Western Australia

Nedlands

WA 6907

Australia

Keywords: Airway smooth muscle bronchial morphology

bronchial responsiveness

Received: June 131995

Accepted after revision November 211995

This work was supported by the National Health and Medical Research Council of Australia.
Smooth muscle contraction results in airway narrowing, but the location of narrowing within the tracheobronchial tree in diseases such as bronchial asthma is unknown. Efforts to measure airway narrowing in intact lungs have included tantalum bronchography [1,2], ultrasonic imaging [3], and high resolution computed tomography $[4,5]$. Results from these studies, and others, suggest that airway narrowing responses are not uniform throughout the bronchial tree. MCNAMARA et al. [4] reported that, in excised intact dog lungs challenged with carbachol, the degree of narrowing differed among airways of different sizes, with narrowing being greater in intermediate-sized airways and less in the smallest and largest airways. Similar findings were reported by CABEZAS et al. [2] from in vivo studies in dogs.

Recently Mitchell and SparRow [6] used perfused porcine bronchial segments to establish the narrowing characteristics of individual airways of various sizes in vitro, and found a greater than 2 -log difference in acetylcholine (ACh) sensitivity between the largest and smallest diameter segments examined. This was the first time that intact bronchial segments, rather than isolated smooth muscle strips or bronchial rings, had been used to study the responsiveness of different sized airways, and such size-related sensitivity differences had not previously been reported.
Factors which may affect airway narrowing at different sites in vitro and in vivo include inner wall area [7, 8], drug distribution and access to the smooth muscle via the epithelium [6,9-12] as well as smooth muscle sensitivity and force production. Furthermore, in a dynamic in vitro model, such as the perfused bronchial segment, responsiveness at different anatomical locations could be influenced by the degree of wall movement and lumen diameter changes occurring in the same segment during narrowing, or by variable ACh concentration gradients within the airway wall as a consequence of variations in the rate of fluid flow through the segment lumen. In the intact airway, the amount of smooth muscle, and its orientation in the airway wall may affect airway responsiveness by determining the extent of shortening of the smooth muscle against the elastic loads present in the airway wall [13-15].

This study was designed to determine whether the responsiveness of large and small airways to cholinergic stimulation in vitro is related to differences in force generating capacity and sensitivity of the smooth muscle in different sized airways. This was examined directly, using bronchial rings, and indirectly, by measuring intralumenal pressure generated by isovolumic bronchial segments. In the pig lung, sensitivity to carbachol and stress generated by smooth muscle strips are very similar within 
the trachea and large airways [16], but these smooth muscle properties have not been systematically studied throughout the bronchial tree using airway segments and rings. The absence of flow or wall movement in the isovolumic segments also allowed us to determine whether variable flow, wall movement, or agonist concentration gradients contributed to the size-related sensitivity differences previously measured in perfused bronchial segments.

\section{Methods}

\section{Tissue preparation}

Lungs were obtained from eight pigs $(25-30 \mathrm{~kg}$ ) within $10 \mathrm{~min}$ of euthanasia. They were placed on ice and taken to the laboratory, where the right lower lobe stem bronchus was dissected free of surrounding lung parenchyma from just below its junction with the right upper bronchus to within $0.5 \mathrm{~cm}$ of the lobe margin. Side branches were ligated close to the wall of the stem bronchus and the bronchus was placed in iced Krebs solution (mmol.L $\mathrm{L}^{-1}: \mathrm{NaCl} 121, \mathrm{KCl} 5.4, \mathrm{MgSO}_{4} 1.2, \mathrm{NaH}_{2} \mathrm{PO}_{4} 1.2$, $\mathrm{NaHCO}_{3} 25$, glucose 11.5 and $\mathrm{CaCl}_{2} 2.5$ ).

\section{Bronchial segments}

The stem bronchus was divided into four $15-20 \mathrm{~mm}$ segments, which were classified as upper, middle-upper, middle-lower and lower, based on their position relative to the tracheal bifurcation. A $4 \mathrm{~mm}$ length of bronchus was left between the proximal end of one segment and the distal end of the next largest to ensure that the segments were not contiguous. Internal diameters were estimated using machined metal rods inserted just inside the proximal and distal end of each segment. The proximal and distal values were then averaged to obtain an internal diameter value for each segment. The range of mean internal diameters for the four segment categories were $5.0-6.0,3.0-4.0,1.8-2.8$, and $1.0-1.4 \mathrm{~mm}$, with an overall average of $5.5,3.5,2.5$ and $1.2 \mathrm{~mm}$, respectively, for each classification. Both ends of each bronchial segment were cannulated and the segment was mounted horizontally in a $30 \mathrm{~mL}$ perspex bath containing Krebs solution $\left(37^{\circ} \mathrm{C}, \mathrm{pH} 7.4,95 \% \mathrm{O}_{2}, 5 \% \mathrm{CO}_{2}\right)$. The proximal cannula was connected to a reservoir containing Krebs solution set at a constant pressure head of $6 \mathrm{cmH}_{2} \mathrm{O}$, enabling the segment lumen to be filled with fluid (fig. 1). A pressure head of $6 \mathrm{~cm}$ was on the plateau of the passive pressure/active pressure response curve for all airway sizes studied, as determined in prior experiments. Lumen and bath solutions were replaced every 15 min throughout each experiment. Taps at either end of the bath could be closed off, creating an isovolumic segment in which increased bronchial tone was measured as an increase in intralumenal pressure above baseline. Pressure changes were measured using a pressure transducer (MPX2010GP, Motorola Semiconductors, Phoenix, AZ, USA) continuous with the segment lumen.

Acetylcholine (ACh) sensitivity and maximum pressure response were measured for each segment. ACh was dissolved in Krebs solution for each experiment. Each segment equilibrated for $60 \mathrm{~min}$ and was then stimulated with a submaximal ACh dose $\left(10^{-6}\right.$ to $\left.10^{-4} \mathrm{M}\right)$, followed upon recovery by electrical field stimulation (60 $\mathrm{V}, 2 \mathrm{~ms}, 20 \mathrm{~Hz}$ ) delivered via platinum electrodes placed $1 \mathrm{~cm}$ apart and encircling the segment. A cumulative concentration-response curve was then obtained for ACh added to the adventitial bathing solution. From these data, the maximum effect (Emax), concentration producing half the maximum effect (EC50) and the negative log of the EC50 ( $\left.\mathrm{pD}_{2}\right)$ were calculated. To confirm that the $6 \mathrm{~cm}$ transpulmonary pressure used did not selectively alter the operating length of the airway smooth muscle in different sized segments and affect maximum pressure or sensitivity, a separate study was performed using airways from four pigs. A range of similar sized airway segments were studied at transmural pressures of 3,6 and $12 \mathrm{cmH}_{2} \mathrm{O}$, and maximum pressure and $\mathrm{pD}_{2}$ were calculated for each segment.

In a separate series of experiments, a noncumulative ACh dose-response curve was performed on each segment with increasing concentrations of $\mathrm{ACh}$ added to the adventitial bathing solution. After each agonist dose, the drug was washed out and the tissue allowed to relax
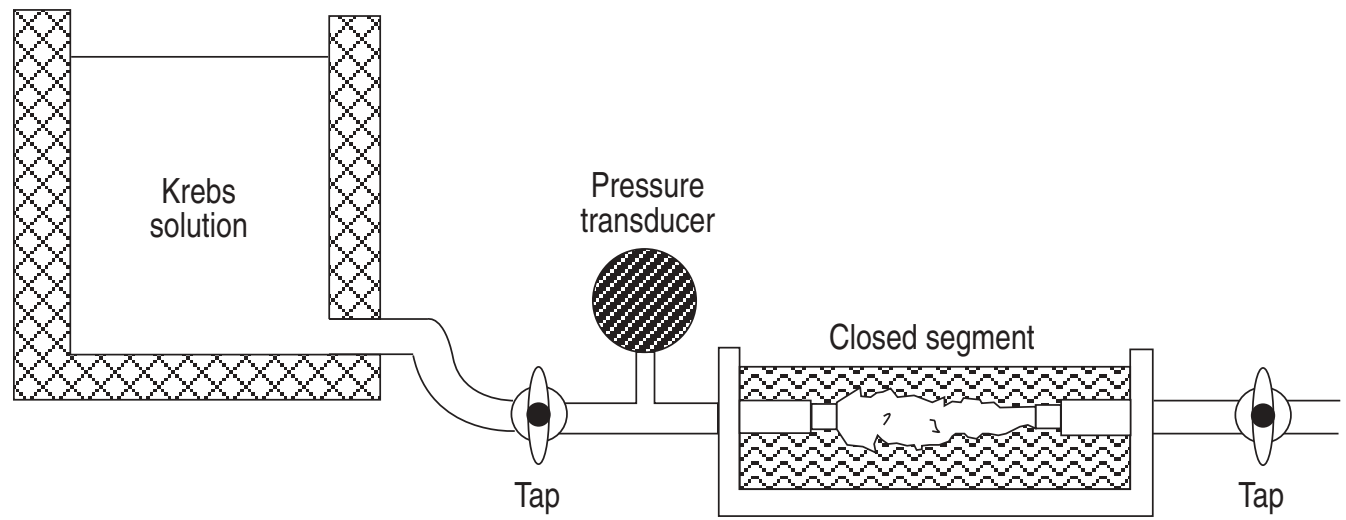

Water-jacketed organ bath

Fig. 1. - Apparatus for measuring pressure developed by an isovolumic bronchial segment. 
for $15 \mathrm{~min}$ prior to the next drug addition. Electrical field stimulation confirmed tissue recovery prior to each dose. Following a 60 min recovery period, the dose response curve was repeated with the adventitial and lumenal surfaces exposed to identical ACh concentrations. This was done in order to minimize any $\mathrm{ACh}$ concentration gradient that may have developed across the airway wall. ACh was introduced into the lumen via the distal tap, while the proximal tap was open. Both taps were then quickly closed and the same concentration of ACh was added to the adventitial bath. The delay between lumenal and adventitial ACh exposure was less than $8 \mathrm{~s}$. The response to each dose was measured at steady-state; when the response had reached a plateau the Emax, EC50 and $\mathrm{pD}_{2}$ were calculated for each curve and compared with the same values from adventitial ACh curves.

\section{Bronchial rings}

Transverse $2.0 \mathrm{~mm}$ wide rings with diameters matching those of the bronchial segments were cut from the right stem bronchus of six animals. Each ring was attached to a pair of hooks in a $10 \mathrm{~mL}$ organ bath. The lower hook was fixed and the upper was connected to an isometric force transducer (Model 7004, Ugo Basile Biological Apparatus, 21025 Comerio-Varese, Italy). The organ bath contained Krebs solution $\left(37^{\circ} \mathrm{C}, 95 \% \mathrm{O}_{2}, 5 \% \mathrm{CO}_{2}\right)$. Active force was recorded on a chart recorder (Model 7D Polygraph, Grass, Quincey, MA, USA). Rings were allowed to equilibrate in the bath for $30 \mathrm{~min}$, then gently stretched to a passive tension of $0.5 \mathrm{~g}$ (determined to be on the plateau phase of the length-tension curve for this range of ring sizes in prior studies utilizing electrical field stimulation to produce active tension). This degree of passive tension caused rings of all sizes to assume a flattened oval configuration between the hooks. Submaximal ACh stimulations $\left(10^{-4} \mathrm{M}\right)$ were performed until repeatable responses were obtained, then a cumulative concentration-response curve to ACh was determined. These data were then used to derive Emax, EC50 and $\mathrm{pD}_{2}$ for each ring.

To compare force generated by rings with pressure generated by bronchial segments, maximum force $(\mathrm{g})$ was divided by longitudinal muscle width $(\mathrm{cm})$ for each ring to obtain tension $\left(\mathrm{g} \cdot \mathrm{cm}^{-1}\right)$. Tension was then divided by ring radius $(\mathrm{cm})$ to obtain a pressure $\left(\mathrm{g} \cdot \mathrm{cm}^{-2}\right)$ and this was converted to $\mathrm{cmH}_{2} \mathrm{O}\left(1.3 \mathrm{cmH}_{2} \mathrm{O}=1.36 \times 10^{-3} \mathrm{~kg} \cdot \mathrm{cm}^{-2}\right)$. Pressure values for individual rings were grouped into diameter ranges corresponding to those used for the segments. These estimated pressures were then compared with the measured pressures from similar sized bronchial segments.

\section{Histology}

At the end of each experiment, individual rings were fixed at optimal length (Lo) using $10 \%$ formalin in phosphate-buffered saline. They were then rinsed in phosphate-buffered saline, embedded (Cryo-M-Bed; Bright,
UK), frozen in isopentane cooled by liquid nitrogen, and $10 \mu \mathrm{m}$ sections were cut with a cryostat. Duplicate sections were stained and the width of the preparation was measured using an image analysis workstation.

\section{Statistics}

Maximum pressure and $\mathrm{pD}_{2}$ values in the bronchial segments from each animal were compared using a repeated measures analysis of variance (ANOVA). Where significant differences were detected, Tukey's test for multiple comparisons was performed. The same analysis was used to compare $\mathrm{pD}_{2}$ among different sized bronchial rings. Results from the simultaneous lumenal/adventitial ACh $v s$ adventitial ACh only were compared using a paired t-test. Results were expressed as mean \pm SEM. Significance was set at a p-value of less than 0.05 .

\section{Results}

\section{Bronchial segments}

Adventitial exposure. There was an upward trend in maximum pressure generation as airway diameter decreased, with pressures generated by the 2.5 and $3.5 \mathrm{~mm}$ groups being significantly greater than those generated by the $5.5 \mathrm{~mm}$ group (fig. 2). This trend was reversed with the smallest airways, where the $1.2 \mathrm{~mm}$ group generated significantly lower pressures than did the 2.5 and $3.5 \mathrm{~mm}$ groups $(\mathrm{p}<0.05)$.

There were consistent differences in the sensitivity of bronchial segments from the same animal to ACh added to the adventitial bathing solution, with the $\mathrm{pD}_{2}$ decreasing significantly with increasing segment diameter (fig. 3 ). There was a significant correlation between $\mathrm{pD}_{2}$ and

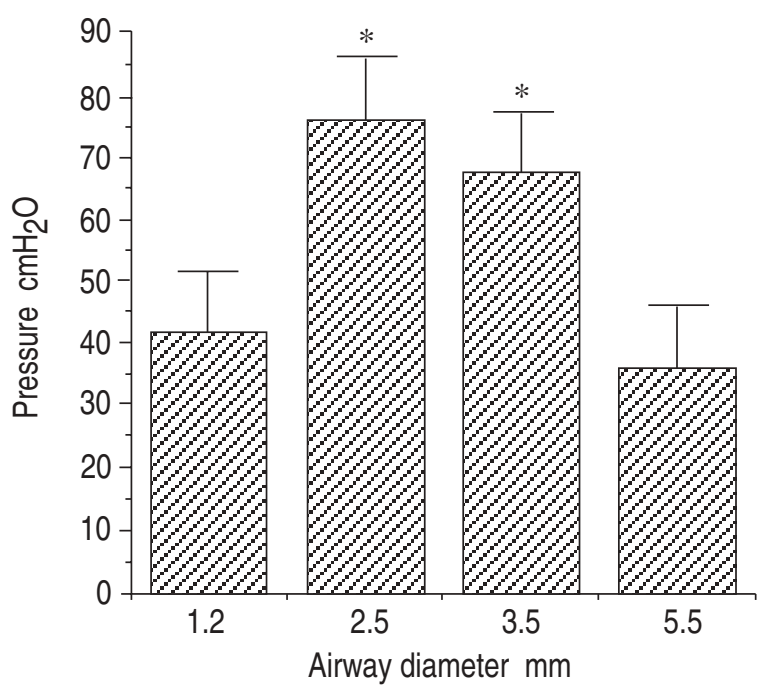

Fig. 2. - Bronchial segment pressure generation $\left(\mathrm{cmH}_{2} \mathrm{O}\right)$ vs segment internal diameter. Values are presented as mean \pm SEM. *: significantly different from 1.2 and $5.5 \mathrm{~mm}$ values $(\mathrm{p}<0.001)$. 


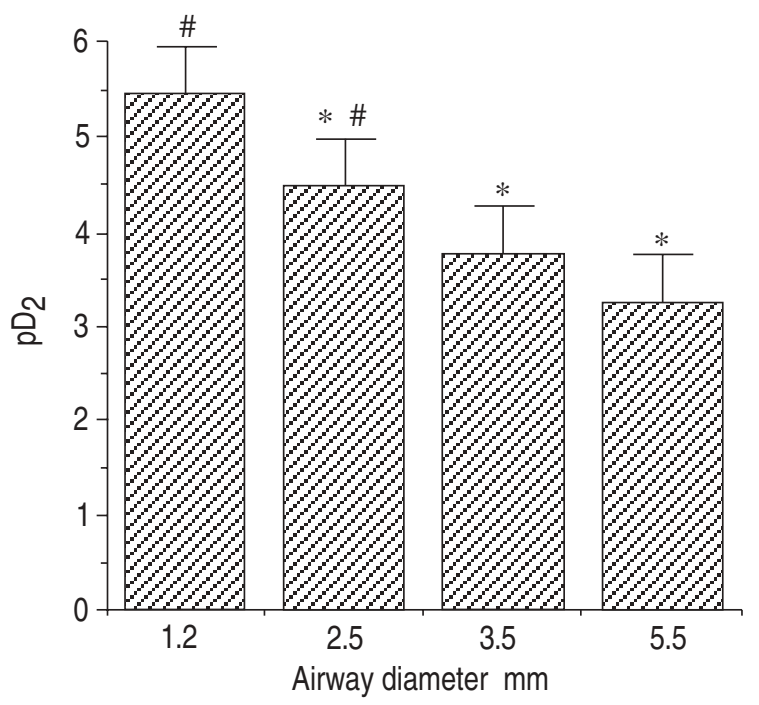

Fig. 3. - Bronchial segment $\mathrm{pD}_{2}$ values for adventitially applied acetylcholine vs segment internal diameter. Values are presented as mean \pm SEM. *: significantly different from $1.2 \mathrm{~mm}$ values $(\mathrm{p}<0.01)$; \#: significantly different from $3.5 \mathrm{~mm}$ values $(\mathrm{p}<0.05) . \mathrm{pD}_{2}$ : negative $\log$ of the concentration producing half the maximal effect (EC50).

diameter $(\mathrm{r}=0.8408 ; \mathrm{p}<0.01)$ (fig. 4). Varying the transmural pressure from 3 to $12 \mathrm{cmH}_{2} \mathrm{O}$ did not affect the pattern of maximum pressure responses or ACh sensitivity (tables 1 and 2).

\section{Simultaneous lumenal and adventitial ACh exposure}

Responses of segments to noncumulatively added ACh were similar to the responses to cumulative ACh measured in the first part of the study, and were unaffected by the route of drug application (simultaneous adventitial and lumenal, or adventitial surface only). There were no significant differences in $\mathrm{pD}_{2}$ or maximum pressure generated for adventitial and lumenal ACh exposure versus adventitial ACh exposure alone.

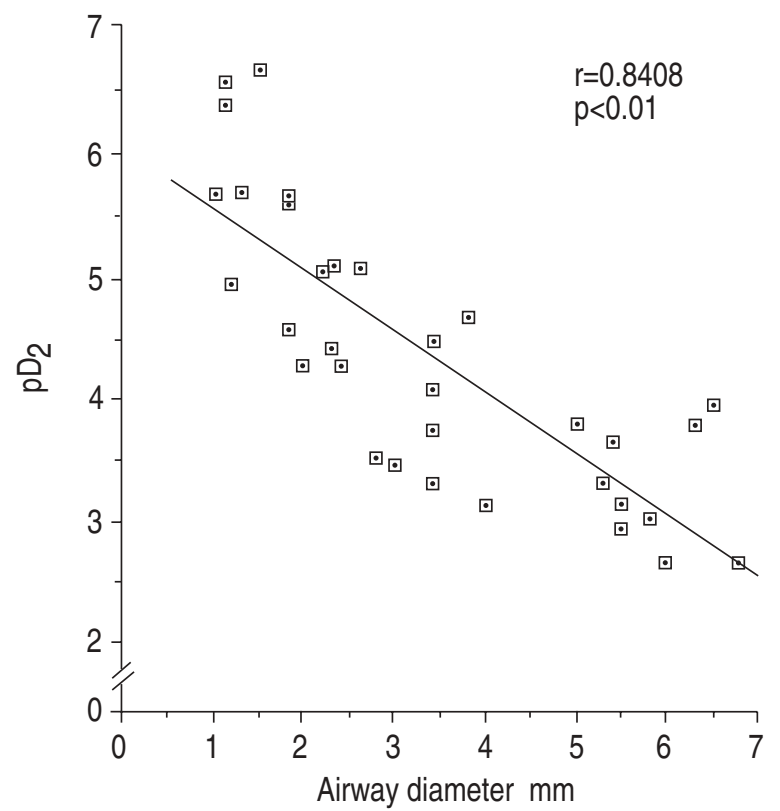

Fig. 4. - Individual bronchial segment $\mathrm{pD}_{2}$ values for adventitially applied acetylcholine $v s$ segment internal diameter.
Table 1. - Effect of transmural pressure on maximum segment pressure $\left(\mathrm{cmH}_{2} \mathrm{O}\right)$ generated in response to adventially applied ACh $(n=4)$

\begin{tabular}{lcrcc}
\hline \multirow{2}{*}{$\begin{array}{l}\text { Transmural } \\
\text { pressure } \\
\mathrm{cmH}_{2} \mathrm{O}\end{array}$} & \multicolumn{5}{c}{ Airway diameter mm } \\
\cline { 2 - 5 } & 1.4 & 2.5 & 4.1 & 5.8 \\
\hline 3.0 & $61.7 \pm 13.8$ & $100.3 \pm 8.0$ & $78.6 \pm 3.9$ & $39.7 \pm 7.8$ \\
6.0 & $65.1 \pm 20.2$ & $98.5 \pm 6.8$ & $77.2 \pm 3.4$ & $42.6 \pm 2.3$ \\
12.0 & $76.5 \pm 18.8$ & $97.4 \pm 6.9$ & $71.8 \pm 6.6$ & $45.0 \pm 3.7$
\end{tabular}

Values are presented as mean \pm SEM. ACh: acetylcholine.

Table 2. - Effect of transmural pressure on segment $\mathrm{pD}_{2}$ values for adventially applied ACh $(n=4)$

\begin{tabular}{lcccc}
\hline \multirow{2}{*}{$\begin{array}{l}\text { Transmural } \\
\text { pressure } \\
\mathrm{cmH}_{2} \mathrm{O}\end{array}$} & \multicolumn{5}{c}{ Airway diameter mm } \\
\cline { 2 - 5 } 3.4 & 2.5 & 4.1 & 5.8 \\
\hline 6.0 & $5.607 \pm 0.052$ & $4.730 \pm 0.369$ & $3.824 \pm 0.232$ & $2.948 \pm 0.151$ \\
12.0 & $5.661 \pm 0.156$ & $4.783 \pm 0.324$ & $4.000 \pm 0.264$ & $3.124 \pm 0.198$ \\
& $5.941 \pm 0.059$ & $4.844 \pm 0.305$ & $4.071 \pm 0.250$ & $3.238 \pm 0.157$
\end{tabular}

Values are presented as mean \pm SEM. $\mathrm{pD}_{2}$ : negative $\log$ of the concentration producing half the maximal effect (EC50); ACh: acetylcholine.

\section{Bronchial rings}

There were no significant differences in $\mathrm{pD}_{2}$ among different sized rings. Values for rings with average internal diameters $(\mathrm{mm})$ of $1.1,1.9,2.9,3.9$ and 5.2 were $5.020 \pm 0.228,4.990 \pm 0.236,5.200 \pm 0.169,4.770 \pm 0.218$ and $4.340 \pm 0.324$, respectively. Maximum force generated (Emax) in response to $10^{-2} \mathrm{ACh}$ increased steadily with increasing ring diameter from $1.21 \mathrm{~g}$ in $1.0-1.5 \mathrm{~mm}$ rings to $4.29 \mathrm{~g}$ in $5.5-7.0 \mathrm{~mm}$ rings. This increase in force corresponded to a threefold increase in smooth muscle cross-sectional area previously measured over this range of ring diameters (unpublished data). Theoretical ring pressures matched measured segment pressures for all but the smallest segments (fig. 5)

\section{Discussion}

In this study, we demonstrated a relationship between airway diameter and smooth muscle force generation (Emax), measured indirectly as a pressure increase in isovolumic bronchial segments. The upward trend in pressure generated from large to small diameter airways could be predicted, in part, by Laplace's Law, which states that for a cylinder with a single surface, pressure is inversely proportional to the radius $(P=\mathrm{T} / \mathrm{r})$. Tensions produced by bronchial rings over the size range covered by the bronchial segments had been measured, and so these tensions were used plus the measured diameter to model the behaviour of the bronchial segments (fig. 5). The behaviour of 5.5, 3.5 and $2.5 \mathrm{~mm}$ groups fitted this model well, where the upward pressure trend accompanying decreasing diameter reflected the greater relative contribution of 


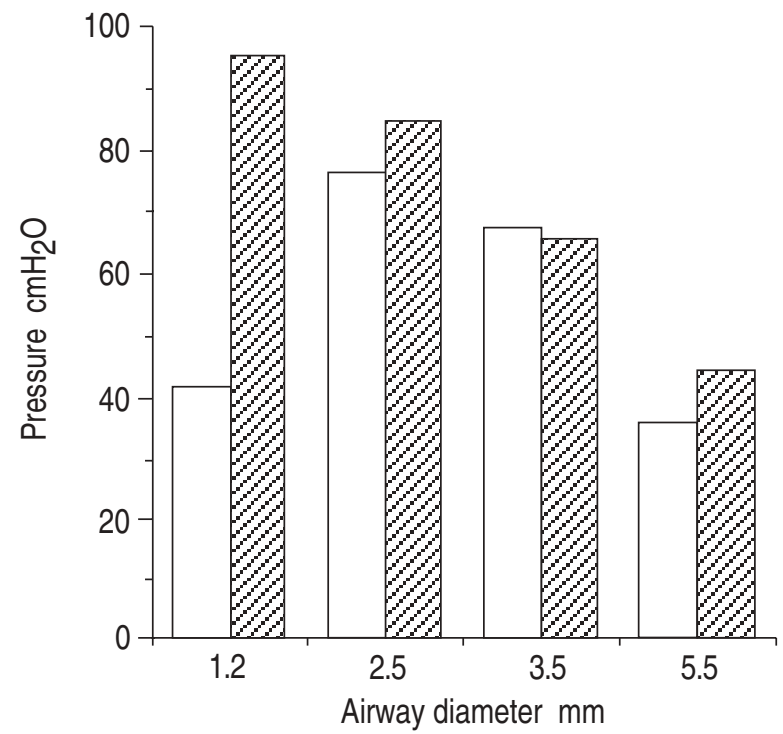

Fig. 5. - Measured segment pressures $\left(\mathrm{cmH}_{2} \mathrm{O}\right)$ compared to theoretical pressures calculated by substituting airway diameter $(\mathrm{mm})$ and ring tension $\left(\mathrm{g} \cdot \mathrm{cm}^{-1}\right)$ values in the Laplace equation. $\square$ : measured segment pressure; $\square$ : calculated segment pressure.

radius changes to pressure generation in airway segments. However, the $1.2 \mathrm{~mm}$ group did not follow this trend, with predicted pressures greatly exceeding measured pressures. These smallest diameter airway segments did not behave as if they were composed of a series of successive bronchial rings, where smooth muscle tension development occurs in a circumferential direction only. It is possible that some of the tension developed by muscle fibres in these small airways could be directed longitudinally rather than circumferentially. BATES and MARTIN [17] have constructed a theoretical model of the effects of smooth muscle orientation on bronchoconstriction, and their results suggest that variations in the three-dimensional orientation of smooth muscle around an airway could have a dramatic effect on its behaviour. Such variation has been reported to occur within the wall of different sized human airways. EBINA et al. [13] found that in a subsegmental bronchus, bundles of smooth muscle were arranged relatively densely and along a direction perpendicular to the bronchial axis, whilst muscle bundles in a smaller membranous bronchiole formed a loose network, with most bundles following a spiral course inclined at about $30^{\circ}$ against the cross-sectional plane of the bronchiole.

Our results for in vitro force generation predict that intermediate-sized airways (1.8-4 mm diameter) would have the greatest potential for narrowing in response to $\mathrm{ACh}$, but this does not factor in the tethering action of lung parenchyma, which could act to modify the extent of airway narrowing throughout the bronchial tree. However, our prediction, based on direct measurements, is consistent with the results presented by McNAMARA et al. [4] and CABEZAS et al. [2] using whole lungs. MCNAMARA et al. [4] reported that, in excised canine lungs, maximum airway narrowing in response to carbachol was greater in intermediate-sized airways than in small or large airways, whilst CABEZAS et al. [2] showed that in vivo airway narrowing in response to maximal vagal stimulation was greatest in 1-5 mm diameter airways and less in airways with diameters larger or smaller than this [2]. By using maximal stimuli, these two studies provided information on the ability of different sized airways to narrow, but neither provided information on the relative sensitivity of different sized airways to the same agonist.

In this study, we also demonstrated a clear relationship between airway size and sensitivity to acetylcholine. There was a significant correlation between airway diameter and $\mathrm{pD}_{2}$, which translated to an approximately 160 fold difference in responsiveness between the 1.2 and $5.5 \mathrm{~mm}$ diameter groups. These results are consistent with those reported by Mitchell and Sparrow [6] for porcine airways studied using a perfused bronchial segment system. They found that airways with internal diameters of $1.0-6.0 \mathrm{~mm}$, had $\mathrm{pD}_{2}$ values for $\mathrm{ACh}$ that ranged from 6.25-3.31, which are comparable with those that we obtained (5.58-3.25) for a slightly narrower range of airway sizes using a different system.

The increase in sensitivity with decreasing segment diameter in this study is difficult to explain, since bronchial rings obtained from the same mainstem bronchus showed no such effect. Variations in airway reactivity in vivo have been attributed to differences in aerosol deposition, receptor distribution or airway wall mechanics. Mitchell and SparRow [6] suggested that, in the perfused bronchial segment, tension generated by the smooth muscle could produce greater muscle shortening in small airways and greater narrowing sensitivity, and that the decreased lumen size and increased wall thickness of the small-bore airway amplified narrowing responsiveness. Such an explanation cannot be used to explain our results, since we were working with a fluid-filled isovolumic preparation, where muscle shortening and airway narrowing did not occur. Similarly, variations in receptor distribution cannot explain the differences since, if this were the case, bronchial rings should have exhibited a response pattern similar to that seen with bronchial segments.

One possible explanation is that the ability of adventitially applied ACh to reach the smooth muscle was decreased in the larger bronchial segments, since the adventitial-smooth muscle diffusion distance doubles from small to large segments [6]. Such an increase in diffusion distance could be expected to affect the response time for a segment, but for it to affect responsiveness would require a decrease in the amount of ACh actually reaching the muscle. A steeper adventitia-airway lumen $\mathrm{ACh}$ concentration gradient in large airway segments could achieve this, but the results of the simultaneous lumenal/adventitial ACh applications and the fact that adventitial drug applications occurred under conditions near equilibrium would argue against this. Nonspecific binding to tissues within the airway wall, or breakdown of ACh prior to reaching the muscle could have occurred, but given the similar histological appearance of large and small airways previously reported [6], it is difficult to envisage how a doubling of the diffusion distance alone could result in a 160 fold decrease in sensitivity. 
Alternatively, release of some relaxant factor able to oppose the action of ACh could explain our results, if it were produced in greater quantities in large diameter airways. The epithelium is one potential source for such a substance [18-21], as are the subepithelial tissues [22]. Heterogeneous responses to ACh have been reported to occur in canine [20] and porcine [21] bronchial rings following epithelial removal, but this does not appear to be the case in perfused segments up to $3 \mathrm{~mm}$ in internal diameter [23].

In this study, we have shown that the pressures generated by airway segments in vitro result from a balance between airway radius and the tension developed by airway smooth muscle, as predicted by Laplace. The pressure increase associated with decreasing airway diameter suggests that geometric factors resulting from the reduction in diameter more than balance the decreased smooth muscle tension in all but the smallest airways examined. We have also confirmed the increase in ACh sensitivity with decreasing airway diameter first reported by Mitchell and SpARRow [6] using a different experimental system. These in vitro patterns of agonist sensitivity and force generation may help to explain the pattern of airway narrowing responses reported to occur in vivo [2, 4]. The differences in the relationship between diameter and pressure generation/ACh sensitivity in bronchial segments versus bronchial rings suggest that the sensitivity of airway smooth muscle is a poor predictor of airway sensitivity, and that disrupting the three-dimensional structure of the airway alters in vitro airway responses, particularly in small airways. If this is so, then it is an important factor that should be considered when extrapolating the results of in vitro airway studies to the in vivo situation.

Acknowledgements: The authors would like thank J. Lemon for her technical assistance.

\section{References}

1. Shioya T, Munoz NM, Leff AR. Effect of resting smooth muscle length on contractile response in resistance airways. J Appl Physiol 1987; 62: 711-717.

2. Cabezas GA, Graf PD, Nadel JA. Sympathetic versus parasympathetic nervous regulation of airways in dogs. J Appl Physiol 1971; 31: 651-656.

3. Iizuka K, Dobashi K, Houjou S, Sakai H, Itoh K, Nakazawa T. Evaluation of airway smooth muscle contractions in vitro by high frequency ultrasonic imaging. Chest 1992; 102: 1251-1257.

4. McNamara AE, Muller NL, Okazawa M, Arntorp J, Wiggs BR, Paré PD. Airway narrowing in excised canine lungs measured by high resolution computed tomography. J Appl Physiol 1992; 73: 307-316.

5. Brown RH, Herold CJ, Hirshman CA, Zerhouni EA, Mitzner W. In vivo measurements of airway reactivity using high resolution computed tomography. Am Rev Respir Dis 1991; 144: 208-212.
6. Mitchell HW, Sparrow MP. Increased responsiveness to cholinergic stimulation of small compared to large diameter cartilaginous bronchi. Eur Respir J 1994; 7: 298-305.

7. Wiggs BR, Moreno R, Hogg JC, Hilliam C, Paré PD. A model of the mechanics of airway narrowing. J Appl Physiol 1990; 69: 849-860.

8. Wiggs BR, Bosken C, Paré PD, James A, Hogg JC. A model of airway narrowing in asthma and in chronic obstructive pulmonary disease. Am Rev Respir Dis 1992; 145: 1251-1258.

9. Ilowite JS, Bennett WD, Sheet ZMS, Groth ML, Nierman DM. Permeability of the bronchial mucosa to $99 \mathrm{~m}$ TcDPTA in asthma. Am Rev Respir Dis 1989; 139: 1139-1143.

10. Mitchell HW, Fisher JT, Sparrow MP. The integrity of the epithelium is a major determinant of the responsiveness of the dog bronchial segment to mucosal provocation. Pulm Pharmacol 1993; 6: 263-268.

11. Sparrow MP, Mitchell HW. Modulation by the epithelium of the extent of bronchial narrowing produced by substances perfused through the lumen. Br J Pharmacol 1991; 103: 1160-1164.

12. Omari TI, Sparrow MP, Mitchell HW. Responsiveness of human isolated bronchial segments and its relationship to epithelial loss. Br J Clin Pharmacol 1993; 35: 357-365.

13. Ebina M, Yaegashi $\mathrm{H}$, Takahashi $\mathrm{T}$, Motomiya $\mathrm{M}$, Tanemura M. Distribution of smooth muscles along the bronchial tree: a morphometric study of ordinary autopsy lungs. Am Rev Respir Dis 1990; 141: 1322-1326.

14. Lambert RK, Wiggs BR, Kuwano K, Hogg JC, Paré PD. Functional significance of increased airway smooth muscle in asthma and COPD. J Appl Physiol 1993; 74: 2771-2781.

15. Ishida K, Paré PD, Blogg T, Schellenberg RR. Effects of elastic loading on porcine trachealis muscle mechanics. J Appl Physiol 1990; 69: 1033-1039.

16. Sparrow MP, Mitchell HW. Contraction of smooth muscle of pig airway tissues from before birth to maturity. J Appl Physiol 1990; 68: 468-477.

17. Bates JHT, Martin JG. A theoretical study of the effect of airway smooth muscle orientation on bronchoconstriction. J Appl Physiol 1990; 69: 995-1001.

18. Flavahan NA, Aarhus LL, Rimele TJ, Vanhoutte PM. Respiratory epithelium inhibits bronchial smooth muscle tone. J Appl Physiol 1985; 58: 834-838.

19. Morrison KJ, Yuansheng G, Vanhoutte PM. Epithelial modulation of airway smooth muscle. Am J Physiol 1990; 258: L254-262.

20. Stuart-Smith K, Vanhoutte PM. Heterogeneity in the effects of epithelial removal in the canine bronchial tree. J Appl Physiol 1987; 63: 2510-2515.

21. Stuart-Smith K, Vanhoutte PM. Airway epithelium modulates the responsiveness of porcine bronchial smooth muscle. J Appl Physiol 1988; 65: 721-727.

22. Gray PR, Derksen FJ, Broadstone RV, Robinson NE, Peters-Golden M. Decreased airway mucosal prostaglandin $\mathrm{E}_{2}$ production during airway obstruction in an animal model of asthma. Am Rev Respir Dis 1992; 146: 586591.

23. Sparrow MP, Omari TI, Mitchell HW. The epithelial barrier and airway responsiveness. Can J Physiol Pharmacol 1995; 73: 180-190. 